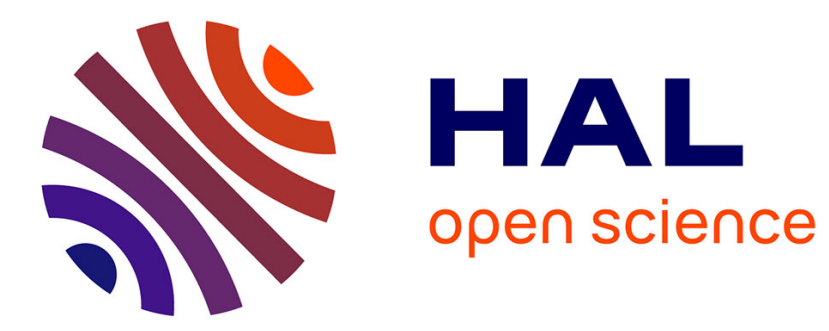

\title{
Three-dimensional dynamics of oblate and prolate capsules in shear flow
}

\author{
Z. Wang, Yi Sui, Peter D.M. Spelt, W. Wang
}

\section{To cite this version:}

Z. Wang, Yi Sui, Peter D.M. Spelt, W. Wang. Three-dimensional dynamics of oblate and prolate capsules in shear flow. Physical Review E : Statistical, Nonlinear, and Soft Matter Physics, 2013, 88, pp.053021. 10.1103/PhysRevE.88.053021 . hal-00931504

\section{HAL Id: hal-00931504 \\ https://hal.science/hal-00931504}

Submitted on 3 Mar 2014

HAL is a multi-disciplinary open access archive for the deposit and dissemination of scientific research documents, whether they are published or not. The documents may come from teaching and research institutions in France or abroad, or from public or private research centers.
L'archive ouverte pluridisciplinaire HAL, est destinée au dépôt et à la diffusion de documents scientifiques de niveau recherche, publiés ou non, émanant des établissements d'enseignement et de recherche français ou étrangers, des laboratoires publics ou privés. 


\title{
Three-dimensional dynamics of oblate and prolate capsules in shear flow
}

\author{
Z. Wang ${ }^{1}$, Y. Sui ${ }^{1}$, P. D. M. Spelt ${ }^{2,3}$, W. Wang ${ }^{1}$ \\ ${ }^{1}$ School of Engineering and Materials Science, Queen Mary University of London, London E1 4NS, UK \\ 2 Laboratoire de la Mécanique des Fluides \& d'Acoustique (LMFA), CNRS, Ecully, France \\ ${ }^{3}$ Département Mécanique, Université Claude Bernard Lyon 1, Villeurbanne, France
}

(Dated: November 5, 2013)

\begin{abstract}
We study computationally the dynamics of oblate and prolate spheroidal capsules in simple shear flow with small inertia for a range of dimensionless shear rates. The capsule is modelled as a liquid droplet enclosed by a hyperelastic membrane, and its equatorial plane is initially tilted out of the plane of shear. We find at low shear rates, the well accepted tumbling motion is not always stable for both oblate and prolate capsules. For an oblate capsule, the dominant stable modes for increasing dimensionless shear rate are: rolling with the equatorial plane staying in the plane of shear, precessing around the vorticity direction, and tumbling. Interestingly, the order of modes is reversed for a prolate capsule: tumbling, precessing and rolling with increasing dimensionless shear rate. At transitional regimes, we find the stable motion of a capsule can depend on its initial titled angle even at the same shear rate. At high dimensionless shear rates, a spheroidal capsule undergoes a complicated oscillating-swinging motion: its major axis oscillates about the plane of shear in addition to the swinging about a mean angle with flow direction found previously, and the amplitudes of both oscillations decrease when increasing the dimensionless shear rate, towards a steady tank treading motion asymptotically. We summarize the results in phase diagrams and discuss the reorientation of both oblate and prolate capsules in a wide range of dimensionless shear rates.
\end{abstract}

\section{INTRODUCTION}

A capsule is a small liquid droplet enclosed by a thin membrane which has shear elasticity. The dynamic motion of a capsule subject to an external flow has been drawing much attention for many years, because it is important for both fundamental research and biomedical or industrial applications (e.g., in micro-encapsulation and drug delivery $[1,2])$. Furthermore, it forms the first step for considering more complicated situations such as capsule suspensions $[3,4]$.

Various types of motion have been identified for a capsule freely suspended in simple shear flow. An initially spherical capsule has always been found to exhibit a steady tank-treading motion, wherein the capsule has deformed into a stationary configuration with a finite inclination with the flow direction and the membrane rotates around the liquid interior (e.g., in Refs [5-8] and a recent review by Barthès-Biesel [9]). The motion of non-spherical capsules is richer in that it is known to yield three modes: a tumbling mode, wherein a capsule flips continuously, a swinging mode, corresponding to periodic shape deformation and small amplitude inclination oscillation with a non-zero mean angle with flow direction when the membrane is rotating around the liquid interior, and a vacillating-breathing mode, in which the capsule's major axis oscillates around the flow direction and the shape shows ample deformation (breathing) $[10,11]$. The vacillating-breathing mode lies between tumbling and swinging. As to which class of motion a non-spherical capsule adopts is known to be affected by the viscosity ratio between the internal and exter- nal liquids [12-14], the membrane viscosity [15-18], the shear rate $[11,19-27]$ and the membrane bending stiffness [28, 29]. In general, the transition from swinging to tumbling can be induced by increasing the viscosity ratio or membrane viscosity, reducing the shear rate, or increasing the membrane bending stiffness when the capsule has a non-spherical reference shape.

All of these modes (tank-treading, swinging, tumbling and vacillating-breathing) are symmetric about the plane of shear, and this symmetry has been imposed (at least on initial conditions) in most previous theoretical and numerical studies on capsules in shear flows. Early experiments by Goldsmith and Marlow [12] and a very recent study by Dupire et al. [30] on red blood cells have suggested the existence of an asymmetrical mode, however. In other systems, such a mode is well documented: a rigid ellipsoidal particle can exhibit a precessing orbit wherein the trajectory of its axis of revolution forms a cone [31], and the angle of the cone depends on the initial inclination angle of the rigid particle. A similar off-theshear-plane motion has been found recently for vesicles in shear flow both theoretically [32] and numerically [3335] and it was named as a 'kayaking' motion [33], which appears when the viscosity ratio and the shear rate are both high. However, a vesicle is different from a capsule in that its membrane is incompressible but does not have any shear elasticity, and such differences in membrane mechanical property lead to very different dynamics in shear flows (e.g., in Refs $[10,34,36,37]$ ). For example, the behaviour of a vesicle is critically determined by the viscosity ratio. At low viscosity ratios a vesicle always undergoes a steady tanking treading motion, indepen- 
dent of the shear rate; however, a non-spherical capsule could have a swinging or a tumbling motion depending on the shear rate at such low viscosity ratios. Without the constraint of membrane shear elasticity, the dynamics of vesicles appears to be richer and recently novel modes such as squaring and parity breaking have been discovered in numerical simulations [35]. So far, the existence of an asymmetrical precessing mode for capsules is still largely an open issue. The only related study is by Dupont et al. [38] on prolate capsules. Clearly there is a need for a numerical study of this possibility for oblate capsules, which forms one motivation of the present paper. In a larger context, it is well accepted that a red blood cell can be modelled mechanically as a liquid-filled oblate capsule (e.g., in Refs [26, 39-41]), so it would be of interest to see to what extent an asymmetrical mode suggested by experimental work cited above would indeed be predicted by this model.

In experiments, yet another mode has been found for red blood cells besides the suggestion of precessing motion discussed above (e.g., in Refs [12, 30, 42]): a cell spins around its axis of revolution with its equatorial planes staying in the plane of shear, like a rolling wheel. These experiments show that this rolling motion can exist under the same conditions as tumbling. It is in general accepted that a red blood cell shares many features in common with an oblate spheroidal capsule, however, such rolling motion has not been reported for oblate capsules with numerical simulation or theoretical approaches, to the best of our knowledge. Only recently with a boundary integral method, Dupont et al. [38] considered the dynamics of prolate spheroidal capsules, whose revolution axis are initially not in the plane of shear. They found that tumbling motion is not stable at low shear rates, and that a prolate capsule will gradually adopt a log-rolling motion with its revolution axis aligned with the vorticity axis.

In summary, for non-spherical capsules in shear flow, with the shear rate increasing, well documented modes are tumbling, vacillating-breathing and swinging; all are symmetric about the plane of shear. Experiments of red blood cells in shear flow have identified an asymmetric off-plane precessing mode and a rolling mode, but these have not been recovered in numerical simulations using the oblate-capsule model, and the relations of these two motions with other modes remain unclear. In this paper, we therefore study the effects of initially tilting a capsule's equatorial plane out of the plane of shear, as an off-plane perturbation, on its subsequent dynamics, seeking specifically to establish the sequence of events when increasing the dimensionless shear rate, which would provide a platform for future theoretical work, as well as future work on suspensions of capsules; also, the work establishes the possibility of coexistence of modes, which has consequences for experimental observations. We use an immersed boundary lattice Boltzmann method [43], which has been well validated for simulating flow-induced deformation of capsules. Both oblate and

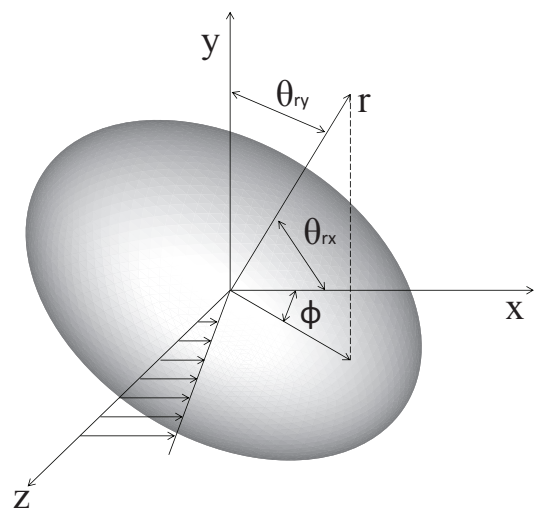

FIG. 1. A spheroidal capsule in shear flow. $\mathbf{r}$ represents the revolution axis. Vorticity axis is the $y$-axis. $\theta_{r y}$ and $\theta_{r x}$ are angles that the capsule's revolution axis makes with the vorticity axis and $x$-axis respectively. $\phi$ is the phase angle defined as the angle that the revolution axis makes with $x$ axis in the plane of shear.

prolate spheroidal capsules are considered. The capsule is modelled as a Newtonian liquid droplet enclosed by a hyperelastic membrane, in the limit wherein the membrane is very thin, hence bending stiffness is neglected. A unity viscosity ratio is used; also, the capsule is assumed to be unstressed at its initial shape. Although real situations are usually more complicated, for example a capsule membrane could be pre-stressed due to osmotic effects, these assumptions form an important limit that has been studied extensively for initial conditions wherein the capsule is symmetric about the plane of shear (e.g., in Refs $[14,24,26])$. Results for a range of initial tilting angles and dimensionless shear rates are categorized (in particular, new regimes for capsules are identified and analysed further) and discussed in light of the above summary of prior work. The sensitivity of the results to the membrane constitutive law used is assessed by comparing results obtained with two different models.

\section{MEMBRANE MODEL AND NUMERICAL METHOD}

We consider an initially spheroidal capsule of various aspect ratios in an incident linear shear flow, $\mathbf{u}=$ $(k z, 0,0)$ where $k$ is the shear rate (Fig. 1). The aspect ratio of the capsule is defined as the length of its revolution axis over that of the equatorial axis, and is thus larger than unity for a prolate capsule and smaller than unity for an oblate capsule. The axis of revolution of the capsule is initially in $\mathrm{y}-\mathrm{z}$ plane and the capsule's equatorial plane is tilted out of shear plane under an angle $\beta$; an equivalent capsule radius is denoted by $a=(3 \mathrm{~V} / 4 \pi)^{1 / 3}$, where $V$ is the volume of the capsule. As stated in the Introduction, some simplifying assumptions are made: the fluids inside and outside the capsule are both taken to be Newtonian and to have the same density $\rho$ and viscosity 
$\mu$, and the capsule membrane is assumed to be very thin, hence bending stiffness is neglected. This leads also to the formation of wrinkles in the simulations that would otherwise be suppressed by bending, but we have found these to remain at acceptable levels (as in related work, e.g., $[7,26])$; also, results are consistent with analytical work, as discussed in Sec. III-IV. Barthès-Biesel and co-workers studied the effect of membrane bending and found that a moderate bending rigidity would alleviate wrinkles but this does not have significant effects on the global deformation and the dynamic motions of a capsule [44].

Results have been obtained for two different membrane constitutive laws in the present study. One is the neoHookean (NH) law, assuming linear dependence of strain energy density with the first invariant of the left CauchyGreen deformation tensor $\lambda_{1}{ }^{2}+\lambda_{2}{ }^{2}+\lambda_{3}{ }^{2}$. The terms $\lambda_{1}, \lambda_{2}$ and $\lambda_{3}$ are the principle extension ratios in the plane of the membrane, and in the direction perpendicular to the plane, respectively. The NH law corresponds to thin membranes made of three-dimensional isotropic and volume-incompressible polymerized materials. The area dilation is unrestricted and is compensated by the thinning of the membrane: $\lambda_{3}=1 / \lambda_{1} \lambda_{2}$. The strain energy function of $\mathrm{NH}$ law for a two-dimensional membrane is given by

$$
W=\frac{1}{6} E\left(I_{1}-1+\frac{1}{I_{2}+1}\right)
$$

where $E$ is the surface shear elasticity modulus, and $I_{1}$ and $I_{2}$ are the first and second strain invariants in two-dimensional, with $I_{1}=\lambda_{1}{ }^{2}+{\lambda_{2}}^{2}-2$ and $I_{2}=$ $\left(\lambda_{1} \lambda_{2}\right)^{2}-1=\left(d A / d A_{0}\right)^{2}-1$. The parameters $d A_{0}$ and $d A$ are initial and final areas of a membrane element. The principal membrane elastic tensions $\tau_{1}$ and $\tau_{2}$, result from $\mathrm{NH}$ law are

$$
\left.\tau_{1}=\frac{E}{3 \lambda_{1} \lambda_{2}}\left(\lambda_{1}^{2}-\frac{1}{\lambda_{1}^{2} \lambda_{2}^{2}}\right) \quad \text { (likewise for } \tau_{2}\right) .
$$

The second constitutive equation used is Skalak's (SK) law [45] to model the membrane of a red blood cell:

$$
W=\frac{1}{6} E\left(\frac{1}{2} I_{1}^{2}+I_{1}-I_{2}\right)+\frac{1}{12} C E I_{2}^{2} .
$$

Postulated in such a way, SK law can capture the special feature of biological membranes that deform easily under shear while almost keep a constant area. For such membranes, on the right hand side of Eq. (3) the factor $C$ (in the second term) must be quite large to ensure negligible area dilation $\left(I_{2} \rightarrow 0\right)$, and the first term mainly accounts for shape deformation. These are clearer when looking at the principle membrane tensions

$$
\tau_{1}=\frac{E \lambda_{1}}{3 \lambda_{2}}\left[\left(\lambda_{1}^{2}-1+C \lambda_{2}^{2} I_{2}\right] \quad \text { (likewise for } \tau_{2}\right) .
$$

We have divided the original strain energy function in [45] by a factor of 1.5 so that Eq. (3) leads to the same small deformation behavior as NH law when $C=1$.
The 3D capsule membrane is discretized into flat triangular elements, and a finite-element membrane model is employed to obtain the forces acting at the discrete nodes of the membrane. The present simulation method is based on the immersed boundary lattice Boltzmann method of Sui et al. [43, 46-48]. The fluid-capsule interaction is solved by the immersed boundary method of Peskin [49], the flow field is solved by the lattice Boltzmann method with a multi-block technique to refine the mesh around the deformable capsule. The method has been validated extensively against results of boundary element simulations and small deformation theory for three-dimensional capsules in shear flow [22, 43]. The simulation set-up (e.g. domain and mesh sizes) in the present study is the same as that in our earlier work, where detailed convergence tests were carried out. For most cases in the present study, the global error in mass conservation of the capsule is within $1.5 \%$.

The following dimensionless groups are used: the Reynolds number is based on the dimension of the capsule, and is defined as $R e=4 \rho k \mathrm{a}^{2} / \mu$; the ratio of viscous and elastic forces is represented as $G=\mu k a / E$, which can be considered to be a dimensionless shear rate or a capillary number. In the present numerical simulations, the shear rate is kept constant and the membrane shear elasticity is varied, so that the Reynolds number does not change but a range of values of dimensionless shear rates can be achieved. We investigate the effect of the Reynolds number on the results towards the end of this paper. The same approach has been used in many previous studies and in such a way the effect of the dimensionless shear rate can be isolated from that of the Reynolds number. In the presentation of the results, the time variable has been made dimensionless with $k^{-1}$.

\section{MOTIONS OF OBLATE CAPSULES}

Results are first presented for an oblate spheroidal capsule with an initial aspect ratio of $2: 3$ and an NH membrane, for increasing the dimensionless shear rate $G$ from $O\left(10^{-4}\right)$ to $O\left(10^{-1}\right)$ whilst keeping the Reynolds number fixed at 0.2. Effects of $R e$ and the initial capsule aspect ratio are discussed further below.

At low dimensionless shear rates, $G \leq 0.01$, it is in general accepted that the capsule will undergo a tumbling motion. In the present study, we find tumbling is not stable to off-plane perturbations; instead, the stable modes of an initially oblate capsule for increasing dimensionless shear rates are: rolling, precessing and tumbling.

At very low dimensionless shear rates, $G \leq 10^{-3}$, the capsule's global deformation is not visible, and it is found that independent of the value of the initial angle $\beta$, the capsule gradually adopts a rolling motion (shown in Fig. 2 and discussed later on). This can be best characterized by the time evolution of the angles that the capsule's revolution axis makes with the $\mathrm{y}$-axis (direction of vorticity) $\theta_{r y}$ (illustrated in Fig. 1) and x-axis (flow direction) $\theta_{r x}$, 


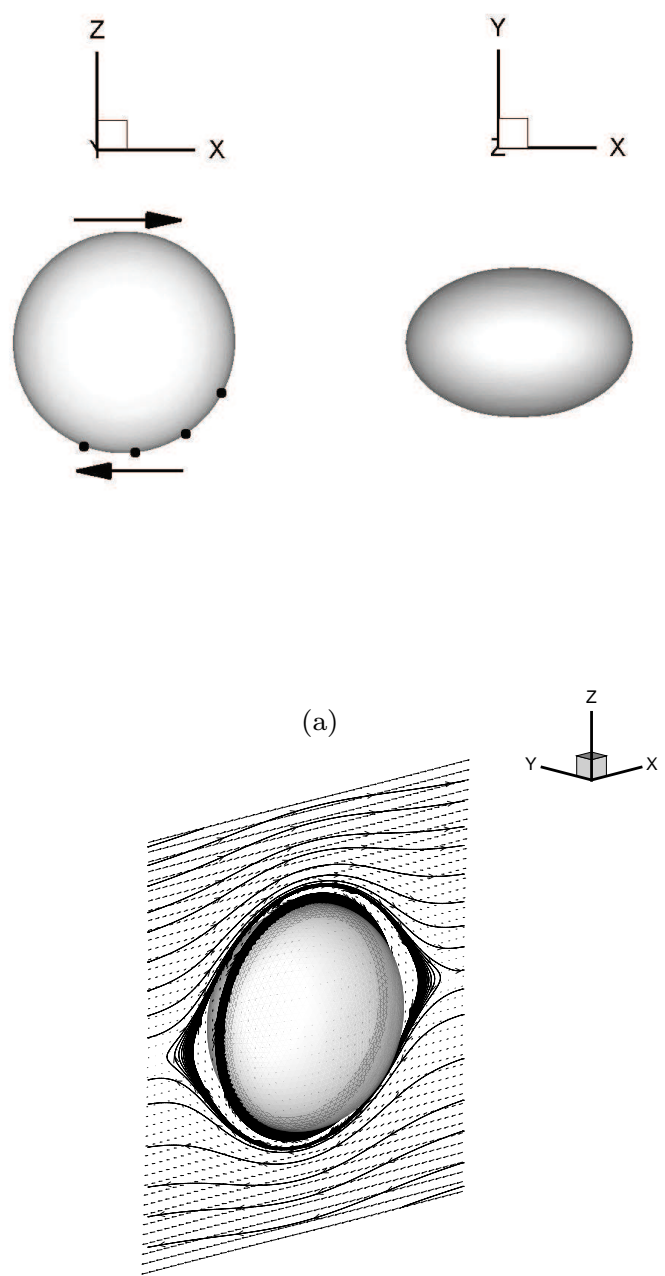

(b)

FIG. 2. Illustration of the rolling motion of an oblate capsule: (a) instantaneous profiles, black dots represent the same membrane node on the capsule's cross section in the plane of shear; (b) flow field around the cross section of the capsule in the plane of shear.

as shown in Fig. 3a for a capsule whose equatorial plane is initially perpendicular to the plane of shear. It is seen that the angle $\theta_{r y}$ decreases in an oscillatory fashion towards zero and that $\theta_{r x}$ exhibits a dampened oscillation around $90^{\circ}$. These results indicate that the motion of the capsule loses its symmetry about the shear plane quickly; a capsule precesses with its equatorial plane gradually becomes aligned with the plane of shear, and then carries out a rolling motion as illustrated in Fig. 2a, in which the capsule spins about its revolution axis like a wheel. From Fig. 2b, it is seen that the capsule membrane in the plane of shear resembles a closed streamline which also suggests a steady configuration. It should be noted that a similar transition to rolling has been observed recently in numerical simulations of rigid oblate spheroidal particles in shear flow with small inertia [50, 51], which we

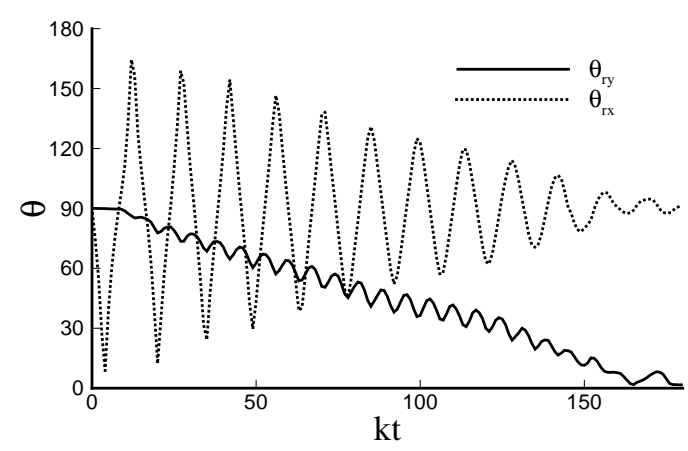

(a)

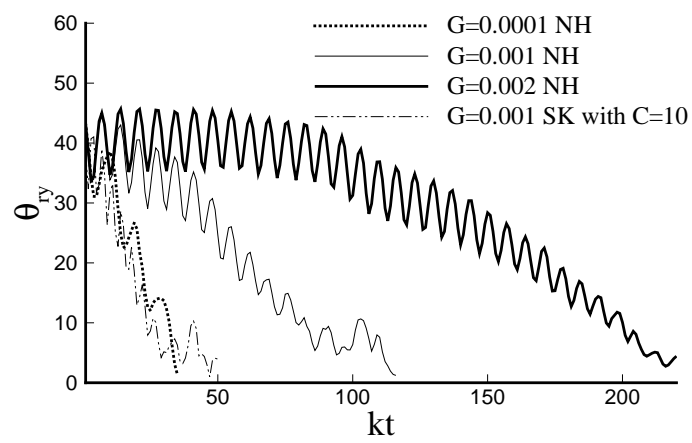

(b)

FIG. 3. Rolling motion of oblate capsules: temporal evolutions of (a) angles that the revolution axis of a capsule with an NH membrane makes with x-axis $\theta_{r x}$, and y-axis $\theta_{r y}$ for $\beta=90^{\circ}$ and $G=0.0005$; (b) $\theta_{r y}$ for capsules with $\mathrm{NH}$ or SK membranes at different dimensionless shear rates and $\beta=45^{\circ}$.

will discuss in Sec. V. The effect of dimensionless shear rate on the time evolution of $\theta_{r y}$ is presented in Fig. 3b. It is seen that at the same initial tilted angle, the dimensionless time it takes for the capsule to change to rolling motion increases monotonically with the shear rate. This transient time also depends on the initial tilted angle, and it is longest for $\beta=90^{\circ}$ for the same dimensionless shear rate. We have also carried out simulations of capsules with an SK membrane and have found a similar transition to rolling motion at low shear rates. An example has been included in Fig. 3b for $C=10$ at $G=0.001$.

When the dimensionless shear rate is increased, a new mode of motion dominates a large range of initial tilted angle values. The oblate spheroidal capsule still largely keeps its initial shape; it carries out a precessing motion and seems to achieve a periodic state after a long transient stage as shown in Fig. 4a. At $G=0.004$, an initial tilted angle of $\beta=22.5^{\circ}$ is not enough to trigger the precessing motion. The equatorial plane of the 
capsule returns to the plane of shear shortly and the capsule finally carries out a rolling motion. For sufficiently large tilted angles, such as $\beta=45^{\circ}$ or $60^{\circ}, \theta_{r y}$ finally oscillates around a constant value which suggests a periodic state. When $G$ is increased slightly to 0.005 , an initial tilted angle of $\beta=22.5^{\circ}$ is sufficient to lead to a precessing motion (Fig. $4 \mathrm{~b}$ and c). Figure $4 \mathrm{~b}$ shows the trajectory of the capsule's revolution axis projected onto a unit sphere. After the transient stage, the revolution axis forms a cone, and its intersection point with the surface of the unit sphere falls into a limit circle.

Apparently similar off-the-shear-plane motion has been described by Jeffery[31] for rigid particles in shear flow. For precessing motion of an ellipsoid of aspect ratio $\chi$ in shear flow without inertia, this corresponds to [31]

$$
\begin{aligned}
\tan \phi & =\chi \tan \left(\frac{k t}{\chi+\chi^{-1}}\right), \\
\tan \theta_{r y} & =\frac{B \chi}{\sqrt{\chi^{2} \cos ^{2} \phi+\sin ^{2} \phi}},
\end{aligned}
$$

where the phase angle $\phi$ is defined as the angle that the revolution axis of the particle makes with the flow direction in the plane of shear (see Fig. 1); $B$ is the so-called orbit constant which only depends on the initial position of release of a rigid particle; its value determines the maximum and minimum values of $\theta_{r y}$ during the periodic precessing motion through

$$
\tan \theta_{r y}^{\min }=B \chi, \quad \tan \theta_{r y}^{\max }=B .
$$

Eq. (7) can be used to fit the value of $B$ to the results of the numerical simulation. In Fig. 4c we show an example comparison of the precessing state with Jeffery's orbit [31] for $\chi=2 / 3$ and a fitted value of $B$. Similar good agreement has been observed for other cases in precessing regime.

We have extracted the time-averaged value and the oscillation amplitude of $\theta_{r y}$ for capsules in the precessing motion; one example is shown in Fig. 5. The results suggest that transition from rolling to precessing happens via a Hopf bifurcation. It is also seen that the oscillation amplitude of $\theta_{r y}$ arrives at a maximum value when the time averaged value is between $30^{\circ}$ and $60^{\circ}$. The time averaged value of $\theta_{r y}$ increases with the dimensionless shear rate in precessing regime, and for sufficiently high dimensionless shear rates and large initial tilted angles, the average value of $\theta_{r y}$ reaches $90^{\circ}$, which corresponds to the axis of revolution of the capsule arriving in the plane of shear and thus a tumbling motion which is symmetric about the plane of shear. Our simulation results show that when $G$ is between 0.01 and 0.02 , the capsule finally carries out the tumbling motion for a wide range of $\beta$. Fig. 6a presents the time series of $\theta_{r y}$ for a capsule with various initial tilted angles. For $\beta=22.5^{\circ}$ and $45^{\circ}$, a tumbling motion is finally achieved, wherein the capsule flips with its shortest axis staying in the plane of shear

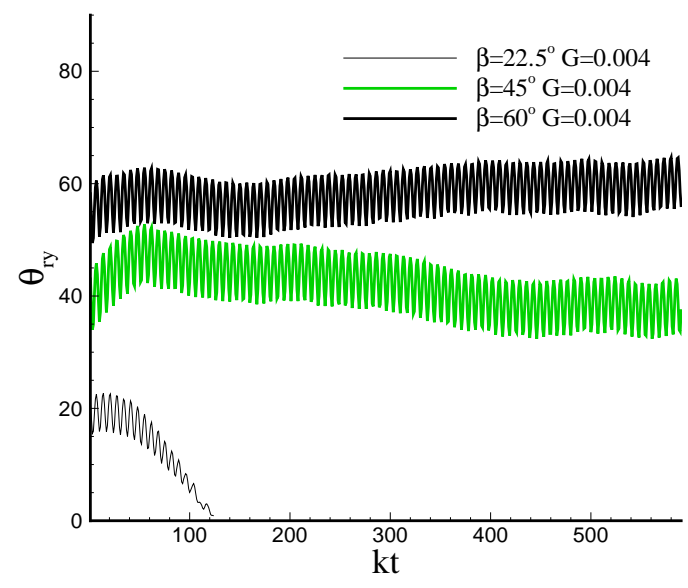

(a)

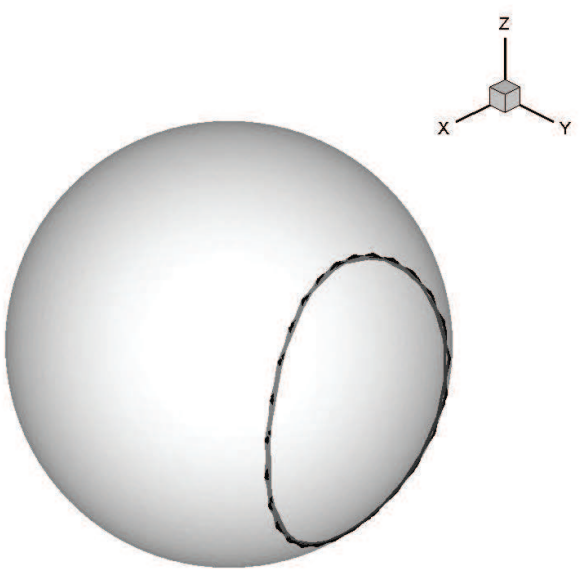

(b)

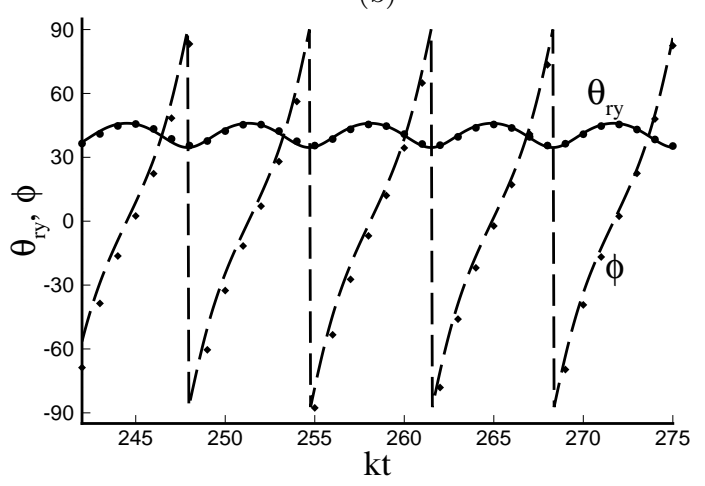

(c)

FIG. 4. (Color online) Precessing motion of an oblate capsule with an NH membrane: (a) temporal evolution of $\theta_{r y}$ of a capsule with various initial tilted angles at $G=0.004$; (b) the stable trajectory of the revolution axis projected to a unit sphere for a capsule at $\beta=22.5^{\circ}$ and $G=0.005$; (c) comparison between numerical simulation (symbols) and Jeffery's theory [31] (curves) in angles $\phi$ and $\theta_{r y}$ for $\beta=22.5^{\circ}$, $G=0.005$. 


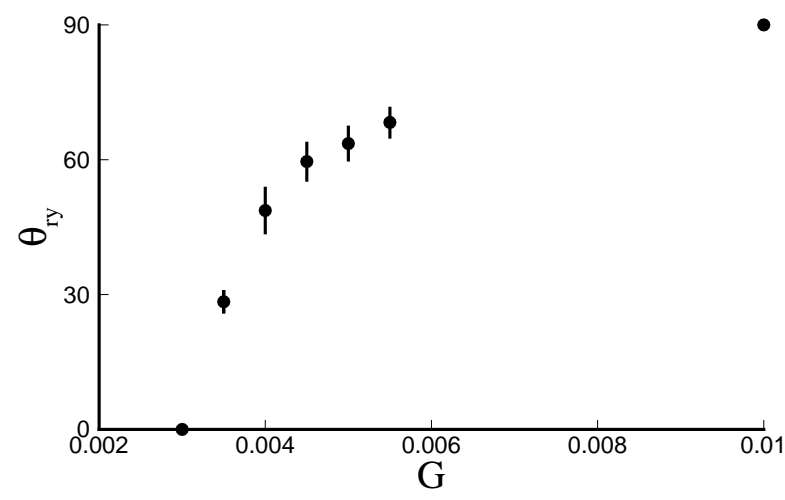

FIG. 5. The time averaged value and the oscillation amplitude of $\theta_{r y}$ as a function of dimensionless shear rate $G$ for an oblate capsule with an $\mathrm{NH}$ membrane and an initial titled angle $\beta=$ $45^{\circ}$.

(see the inset). Interestingly, for $\beta=5^{\circ}$, which can be considered as a small off-the-shear-plane perturbation to a capsule whose equatorial plane is in the plane of shear, the capsule turns back to rolling motion, which suggests rolling motion is strongly stable for an oblate capsule.

At low shear rates $(G \leq 0.02)$, the shape of the capsule largely resembles an oblate spheroid. We can find the length of the revolution axis $c$ and the equivalent diameter of the capsule in its equatorial plane with area $S, b=2(S / \pi)^{0.5}$. A Taylor shape parameter $D=(b-c) /(b+c)$ has been computed and the results are presented in Fig. 6b. It is seen that the oscillation amplitude of the shape parameter increases with $G$ and the minimum value approaches zero. When $D$ becomes close to zero, the projection of the capsule onto the plane of shear is close to a circle and it is difficult to identify a major axis.

The limit $D_{\text {min }} \rightarrow 0$ has been proposed recently in [26] as a criterion for a transition from tumbling to swinging. A swinging regime is indeed achieved when $G$ is beyond 0.03 , where the major axis of the capsule can be identified from the principle axis of the capsule membrane's moment of inertia tensor. In previous studies where the capsule is symmetric about shear plane during deformation (e.g., in Refs $[21,22,26]$ ), it has been found that the angle between the major axis and the flow direction oscillates around a mean value with a small amplitude in a swinging motion. Interestingly, in the present study, we find that initially tilting the capsule out of the shear plane can lead to very complicated swinging states, in which the major axis oscillates about the plane of shear in addition to the previously found swinging about a mean angle with the flow direction. Such an off-plane swinging motion has also been found for prolate capsules in shear flow, and was

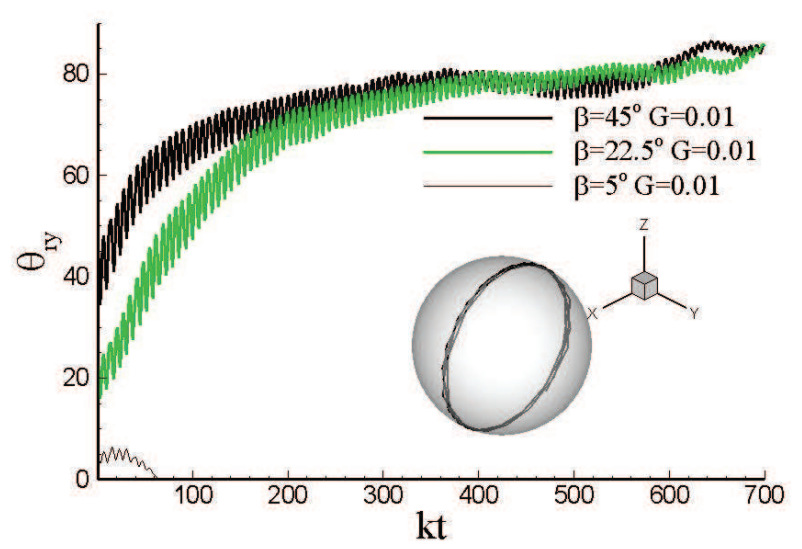

(a)

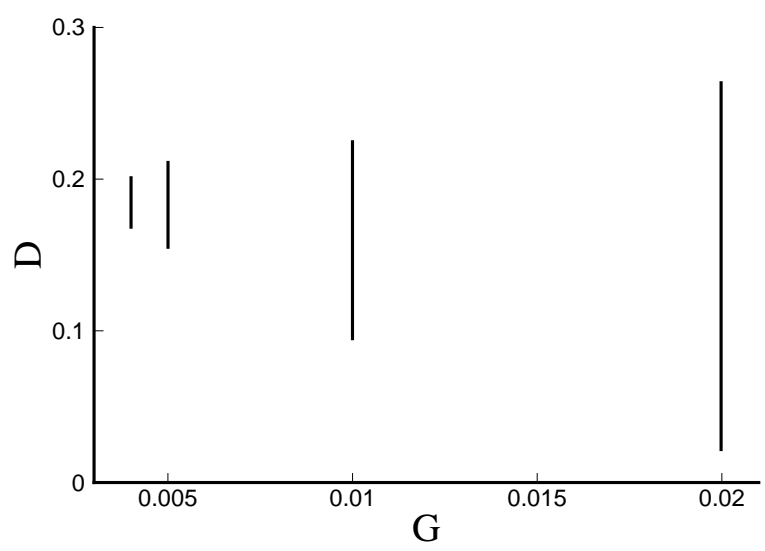

(b)

FIG. 6. (Color online) Tumbling motion of an oblate capsule with an NH membrane: (a) temporal evolution of $\theta_{r y}$ of a capsule at $G=0.01$. The inset shows the stable trajectory of the revolution axis projected to a unit sphere for a capsule with $\beta=22.5^{\circ}$; (b) variations of the Taylor shape parameter at different dimensionless shear rates for a capsule with $\beta=$ $45^{\circ}$.

named as oscillating-swinging (OS) motion [38]; here we use the same name. Figure 7a shows the trajectory of the long axis projected to a unit sphere for such a oscillatingswinging state, and it is seen to form a lemniscate with size decreases when $G$ is increased. Figure $7 \mathrm{~b}$ shows the variations of the angles that the major axis of the capsule makes with the shear plane and $x-y$ plane. When increasing the dimensionless shear rate, both oscillation amplitudes decrease, towards zero which corresponds to a steady tank treading mode. However, there are two exceptions. One corresponds to $\beta=90^{\circ}$, where the capsule carries out a swinging motion whilst adhering to symmetry about the shear plane; this has been well studied 


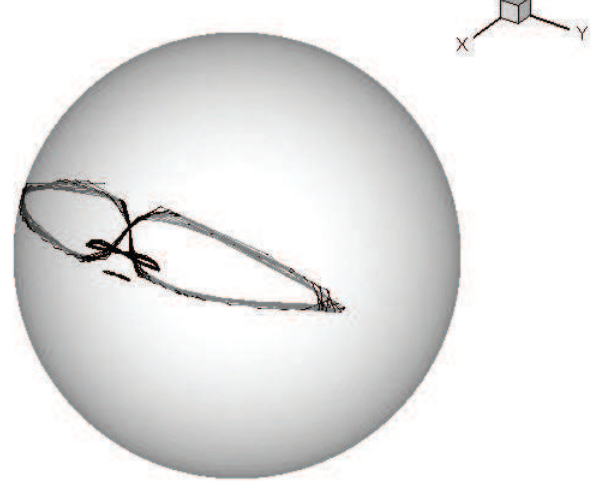

(a)

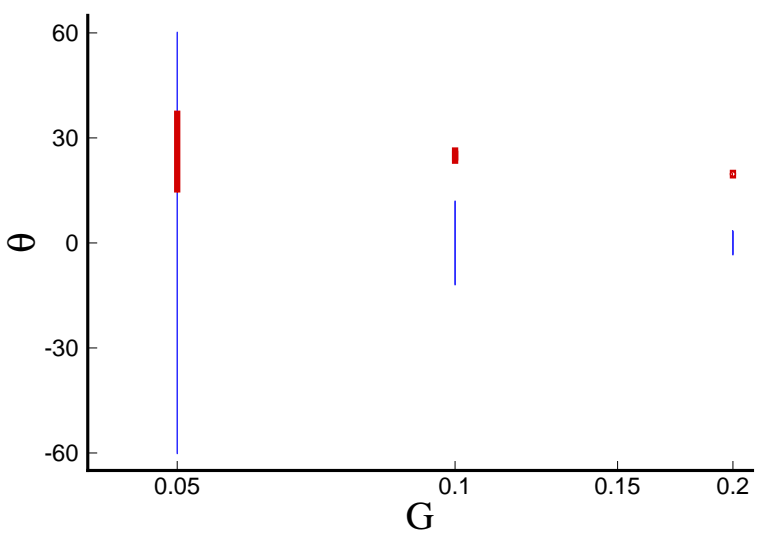

(b)

FIG. 7. (Color online) Oscillating-swinging motion of an oblate capsule with an NH membrane. (a) Trajectories of the long axis of a capsule projected to a unit sphere. The values of $G$ are $0.05,0.1$ and 0.2 respectively with the sizes of the lemniscates decreasing. $\beta=67.5^{\circ}$. (b) Variations of the angle that the long axis of a capsule makes with the plane of shear $\theta_{l x z}$ (blue lines) and the angle that the long axis makes with $x-y$ plane $\theta_{l x y}$ (red bold lines) for increasing dimensionless shear rate.

before. The other exception is for $\beta=0^{\circ}$, i.e., when the capsule's equatorial plane is initially in the plane of shear, where we find the capsule to always be symmetric about the shear plane and this is very stable to small perturbations at $\beta \leq 5^{\circ}$. The capsule achieves a stationary shape with a constant inclination angle with the $x-y$ plane and the membrane rotates around the liquid inside, just like a spherical capsule in shear flow.

Recently, Bagchi and co-workers identified a novel vacillating-breathing mode for non-spherical capsules in shear flow [11], notably when the fluid inside the capsule is much more viscous. This motion happens between tumbling and swinging when increasing the shear rate. Vacillating-breathing mode was first predicted theoret-

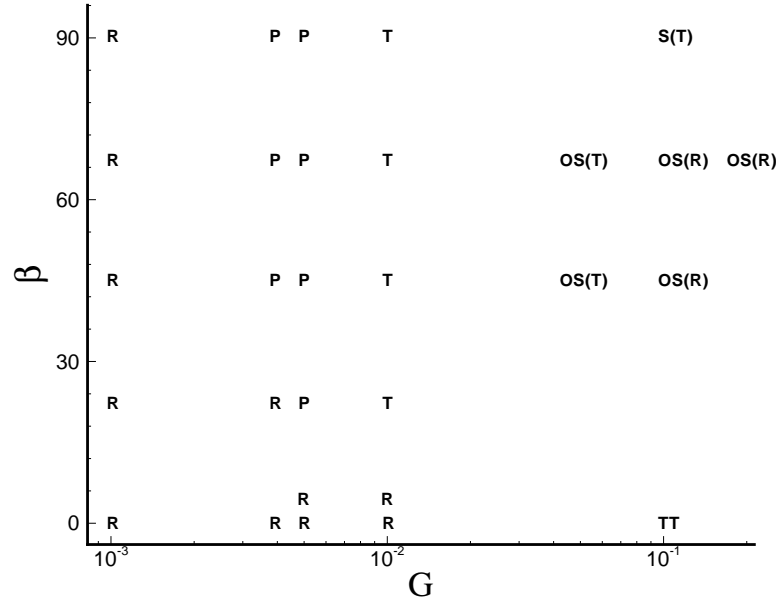

FIG. 8. Phase diagram for an oblate capsule with an initial aspect ratio of $2: 3$ and an $\mathrm{NH}$ membrane. R represents rolling, $\mathrm{P}$ represents precessing, $\mathrm{T}$ is for tumbling, $\mathrm{S}$ for swinging, $\mathrm{OS}(\mathrm{T})$ and $\mathrm{OS}(\mathrm{R})$ are for two types of oscillatingswinging motion as explained in the text, and TT is for steady tank-treading.

ically by Misbah [10] for vesicles. It is different from swinging in that the major axis of the capsule/vesicle oscillates around the flow direction and thus has a zero mean inclination angle. In the present study assuming a unity viscosity ratio, we do find swinging motion with the major axis of the capsule periodically crossing the plane of zero velocity, but the time averaged value of the inclination angle with the flow direction is always considerably larger than zero.

In a recent work by Omori et al. [52] on the dynamics of an oblate spheroidal capsule in shear flow, an interesting reorientation phenomenon was discovered wherein the capsule is in the swinging regime at high shear rates. Defining a unit vector which extends from the mass center of the capsule to the material point of the membrane that initially intersects with the revolution axis of the capsule, it was found that this vector would gradually approach the plane of shear at relatively low shear rates (similar to that in the tumbling motion and here we use OS(T) to represent this mode), and the unit vector would approach the vorticity axis of the shear flow at relatively high shear rates (similar to that in the Rolling motion and here we use $\operatorname{OS}(\mathrm{R})$ to represent this mode). In the present study we observed a similar reorientation for oblate capsules. For example in Fig. 7, $G=0.05$ belongs to the $\mathrm{OS}(\mathrm{T})$ regime and $G=0.1$ and 0.2 belongs to the $\mathrm{OS}(\mathrm{R})$ regime. Detailed features of the reorientation can be found in [52] and are thus not repeated here; such a reorientation will be further discussed in Sec. V, covering both oblate and prolate capsules in a much wider range of dimensionless shear rates.

To summarize the various regimes as a function of the dimensionless shear rate and the initial tilted angle, a phase diagram has been constructed and is shown in Fig. 8, noting that the viscosity ratio is unity and 


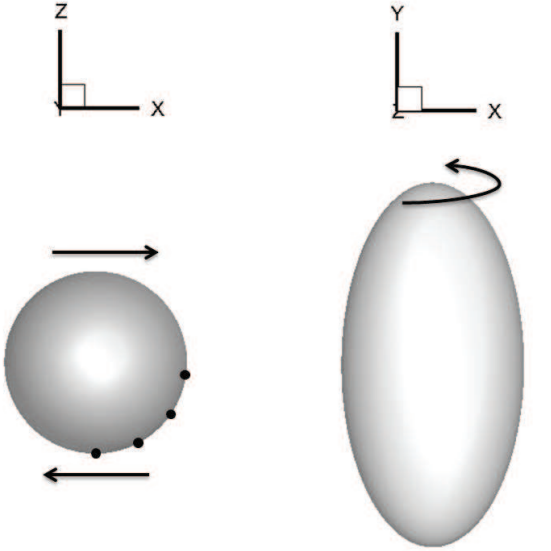

FIG. 9. Illustration of the rolling motion of a prolate capsule. Black dots represent the same membrane node on the capsule's cross section in the plane of shear.

bending has been neglected. Several novel features can be observed from the phase diagram: first, at low dimensionless shear rates ( $G \leq 0.02$ for the present oblate capsule), previous studies assuming an initial symmetry about the shear plane have reported only one tumbling mode, while the present results indicate that when increasing the dimensionless shear rate, the dominant stable motion exhibits the following modes: rolling, precessing and tumbling; second, it is seen that there can be different modes at the same $G$, for example at $G=0.01$, which would explain the coexistence of different modes (e.g., tumbling and rolling) observed in experiments on red blood cells [12]; third, at high dimensionless shear rates, a sufficient initial titled angle could lead to a more complicated oscillating-swinging motion.

\section{MOTIONS OF PROLATE CAPSULES}

In previous studies of prolate capsules in shear flow (e.g., in [26]), the revolution axis of the capsule was initially in the plane of shear. It was found that the capsule undergoes a tumbling motion at low shear rates, and transits to a swinging motion with the shear rate increasing. Recently, Dupont et al. [38] studied for the first time the motions of prolate capsules in shear flow, with the revolution axis being initially titled out of the plane of shear. They found at low dimensionless shear rates, the stable mode is a log-rolling motion, where the long axis of the capsule aligns with the vorticity axis, as illustrated in Fig. 9. They also discovered that with the shear rate increasing the rolling motion becomes unstable, and the capsule precesses around the vorticity axis, which was named as wobbling (illustrated in Fig. 10). At even higher shear rates, the long axis of the prolate capsule tends to approach and to oscillate about the plane of

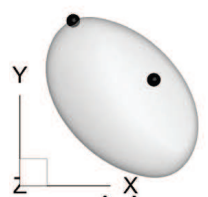

(a)
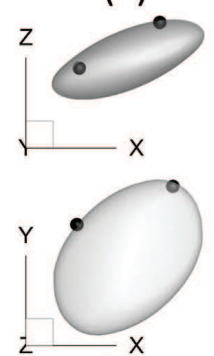

(e)

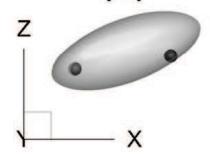

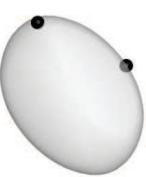

(b)
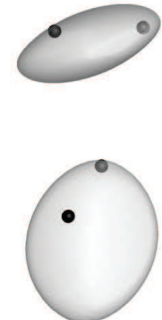

(f)

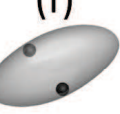

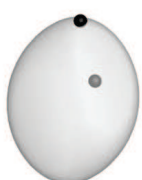

(c)
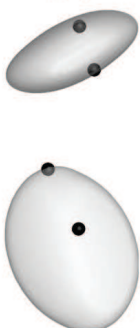

(g)

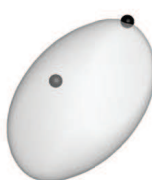

(d)
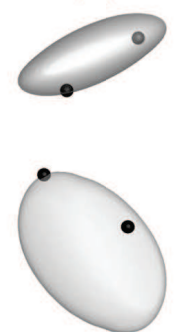

(h)
FIG. 10. Snapshots of a prolate capsule during a wobbling motion. Time sequence is from (a) to (h). Two small spheres are attached to the surface of the capsule as tracer particles.

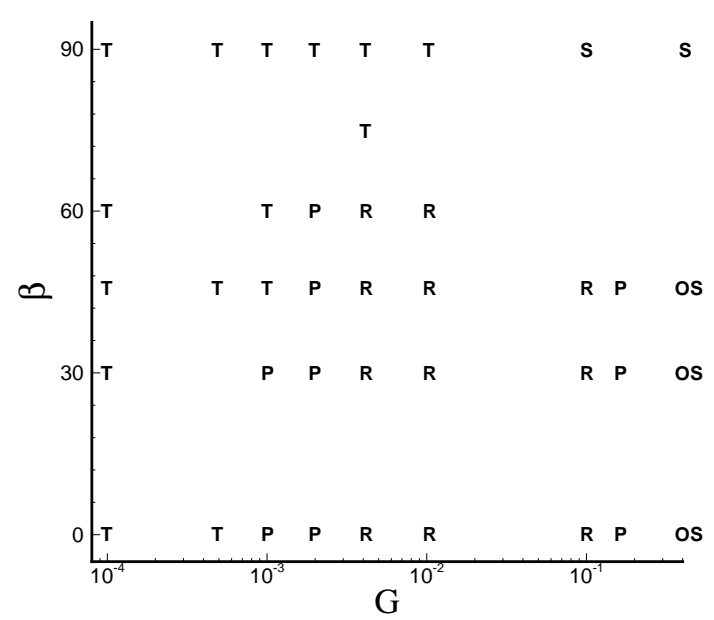

FIG. 11. Phase diagram for a prolate capsule with an initial aspect ratio of $2: 1$ and an SK membrane with $C=1$. T represents tumbling, $\mathrm{P}$ represents precessing, $\mathrm{R}$ is for rolling, $\mathrm{S}$ is for swinging and OS represents oscillating-swinging.

shear, in an oscillating-swinging motion similar to that of an oblate capsule as described in Sec. III. This similarity is possibly because at high shear rates both prolate and oblate capsules deform into elongated ellipsoidal shapes. In the present study, we have also considered the dynamics of prolate spheroidal capsules in shear flow, for situations similar to that of Dupont et al. [38]. The difference between our study and that of Dupont et al. is that we include small inertia, and the lowest dimensionless shear rate considered has been reduced to $G=0.0001$.

In this section, results are presented for a prolate capsule with an initial aspect ratio of 2:1 enclosed by an SK 


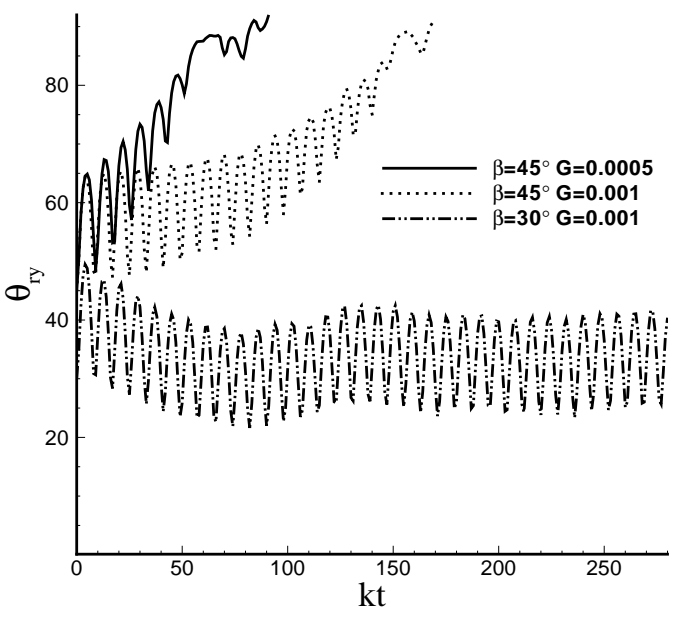

(a)

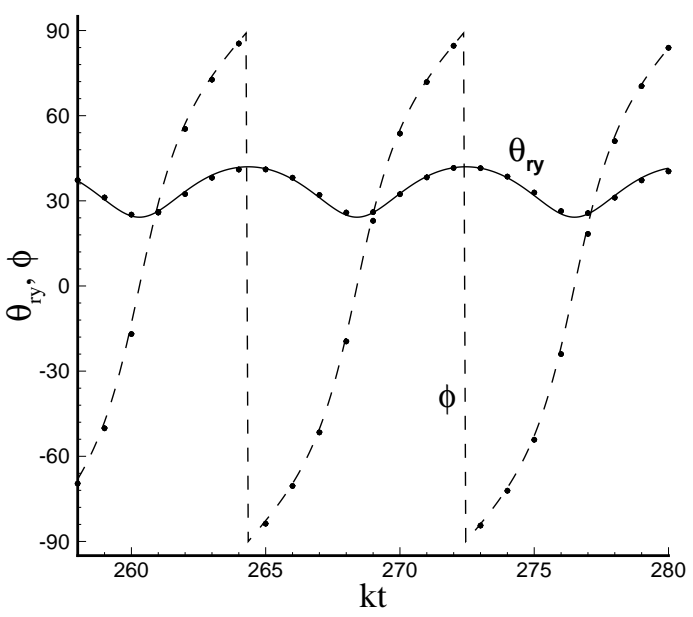

(b)

FIG. 12. (a)Temporal evolution of $\theta_{r y}$ for prolate capsules in tumbling and precessing motions; (b) comparison between numerical simulation (symbols) and Jeffery's theory [31] (curves) in time evolution of angles $\phi$ and $\theta_{r y}$ for $\beta=30^{\circ}$ and $G=0.001$ when a capsule is in a precessing motion.

membrane with $C=1$. The dimensionless shear rate considered ranges from $G=10^{-4}$ to 0.4 and the Reynolds number is kept at 0.2. We summarize the results in a phase diagram as shown in Fig. 11. At relatively high dimensionless shear rates, $G \geq 0.01$, our results are consistent with those of Dupont [38]: with the dimensionless shear rate increasing, we find rolling, wobbling (or precessing) and oscillating-swinging motions as the stable modes. However, at lower dimensionless shear rates, where the deformation of the capsule is very small, we have observed fundamentally new features.

At very low dimensionless shear rates, $G \leq 0.0005$, we find independent of the initial tilted angle a capsule finally adopts a tumbling motion. The capsule places its long axis in the shear plane and rotates about its minor axis. Figure $12 \mathrm{a}$ shows the evolution of $\theta_{r y}$ at low dimensionless shear rates with an initial tilted angel of $45^{\circ}$, it is seen that the transient dimensionless time towards tumbling motion $\left(\theta_{r y}=90^{\circ}\right)$ increases with the dimensionless shear rate. When the dimensionless shear rate is increased to around 0.002 , a precessing motion dominates a wide range of initial tilted angles. In this mode, the long axis of a capsule precesses around the vorticity axis with a nutation and forms a cone. The time evolution of $\theta_{r y}$ of a prolate capsules in a precessing motion is presented in Fig. 12a, and a comparison with Jeffery's orbit [31] is presented in Fig. 12b, where very good agreement is observed. From Fig. 12a, we also infer a dependency of the final stable motion on the initial titled angle, similar to that of an oblate capsule. At $G=0.001$, an initial titled angle of $\beta=45^{\circ}$ leads to a tumbling motion, and $\beta=30^{\circ}$ results in a precessing motion.

In the precessing regime, the time-averaged value of $\theta_{r y}$ decreases with the dimensionless shear rate, towards $0^{\circ}$ which corresponds to a log-rolling motion. For the dimensionless shear rate $G \geq 0.004$, rolling motion is a dominant stable mode where the prolate capsule finally rotates around its long axis which is aligning with the vorticity axis. The time evolutions of $\theta_{r y}$ for prolate capsules in the rolling regime are presented in Fig. 13 for a range of dimensionless shear rates. It is interesting to find that upon increasing the dimensionless shear rate, the transient dimensionless time towards steady rolling first decreases, until approximately $G=0.01$, beyond which it increases until the next stable mode of motion, which is wobbling. We also conclude from Fig. 13 that the tumbling motion of the prolate capsule is strongly stable: initially tilting the revolution axis out of the pane of shear by $15^{\circ}$ is not sufficient to trigger the rolling motion at $G=0.004$, when rolling is the dominant mode. The qualitative difference between the present results and that of Dupont [38] may originate from the inertial effect, which we will discuss in Sec. V.

\section{DISCUSSION}

\section{A. Particle shear stress and effect of Reynolds number}

We investigate the particle shear stress for both oblate and prolate capsules at low shear rates, in order to test the relevance of an hypothesis of Jeffery [31] that nonspherical rigid particles in Stokes shear flow finally adopt a motion with minimum energy dissipation. The normalized particle shear stress is [6]:

$$
\left.\sigma_{x z}=\frac{1}{\mu k V} \int_{A}\left[f_{x} x_{z}+\mu(\lambda-1)\left(u_{x} n_{z}+u_{z} n_{x}\right)\right)\right] d A
$$

where $f_{x}$ is the membrane force in the direction of $x$ and $\lambda$ is the viscosity ratio. The particle shear stress can be 


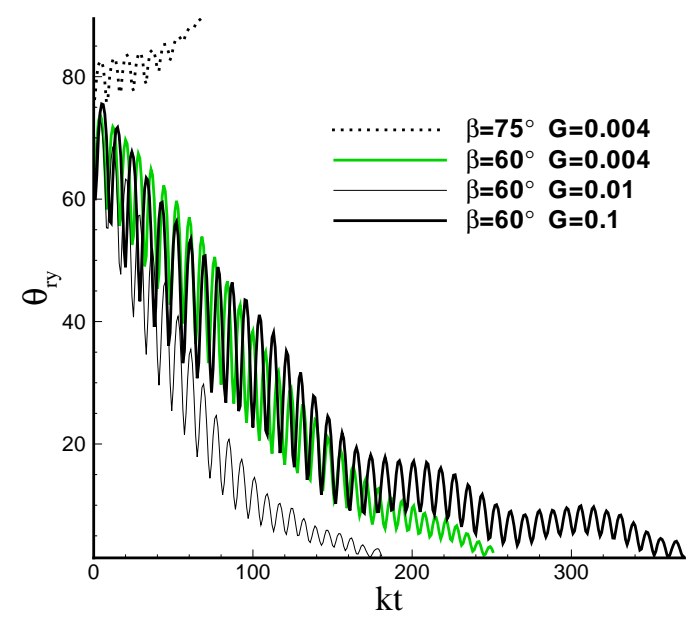

FIG. 13. (Color online) Temporal evolution of $\theta_{r y}$ for prolate capsules that are finally in tumbling and rolling motions.

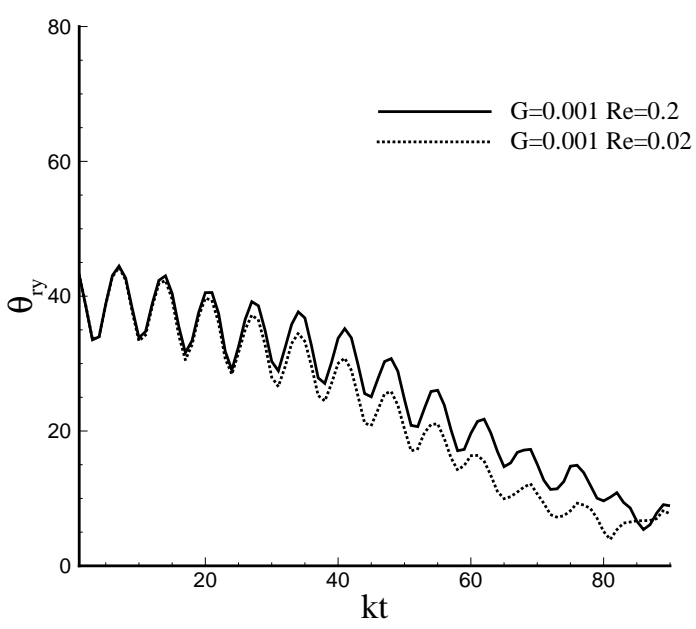

FIG. 14. Temporal evolutions of $\theta_{r y}$ of an oblate capsule with an initial aspect ratio of $2: 3$ and an $\mathrm{NH}$ membrane at $\beta=45^{\circ}, G=0.001$ and $R e=0.02$ and 0.2 .

used to indicate the shear viscosity of the suspension of capsules.

For an oblate capsule with an $\mathrm{NH}$ membrane and an initial aspect ratio of $2: 3$, at $G=0.001$ the value of $\sigma_{x z}$ averaged over one period equals 3.15 for tumbling motion and 3.87 for rolling motion, so it appears the capsule tends to adopt a mode of motion which makes larger contribution to the shear stress. The same phenomenon is observed for prolate capsules, thereby also negating the applicability of Jeffery's hypothesis when extended to flows with finite inertia. However, the solution of the Stokes equations are known to minimize energy dissipation only for given boundary velocities (e.g., in [53]), and the presence of the membrane around the capsule makes the applicability of this theorem here unclear. Furthermore, numerical studies on rigid particles in shear flow $[50,51]$ have shown that when the Reynolds number is within $[0.1,100]$, rigid oblate spheroids tend to carry out a rolling motion and prolate particles tend to tumble, which are very similar to the present findings.

In early experiments of Karnis et al. [54] about axial migration of particles in Poiseuille flow, it was found that inertia becomes sufficient to break the reversibility of the flow when the particle Reynolds number (defined in a similar way to the present study) is of $O\left(10^{-4}\right)$, above which both rigid rods and disks adopt motions that contribute to increasing the suspension viscosity. Drawing an analogy between rigid particles and capsules, we would expect there could be a transition in stable modes of motions for capsules when the Reynolds number is at a similar order. However, reducing Re from the present 0.2 to $10^{-4}$ will increase the computational time by three orders of magnitude, which is currently not accessible. An attempt has been made to assess the significance of inertia here, by carrying out a simulation with reduced $R e$ from 0.2 to 0.02 , for a capsule with an $\mathrm{NH}$ membrane with $\beta=45^{\circ}$ and $G=0.001$. The results as shown in Fig. 14, indicate that the capsule transits to rolling motion in a slightly shorter dimensionless time with decreasing Reynolds number. We therefore leave this issue to future study, when new algorithms that can reduce the computation time to an acceptable level are adopted.

\section{B. Flow decomposition}

In order to understand the mechanism whereby a spheroidal capsule adopts a motion that increases the suspension shear viscosity, we have decomposed the simple shear flow into an elongational flow and a rotational flow for the case presented in Fig. 14 at $R e=0.2$, and studied the dynamics of the capsule in each of these basic flows separately. We have found that the capsule finally undergoes a tumbling motion in the rotational flow; in the elongational flow the capsule achieves a steady state with the revolution axis aligned with the direction of compression and the equatorial plane being perpendicular to the original plane of shear. In both flows, the revolution axis of the capsule is within the original plane of shear, and thus combining these two modes together would naturally lead to a tumbling motion for the oblate capsule. However, the capsule adopts a rolling motion in the combined flow (simple shear flow). This analysis, although does not completely explain the mechanism, it suggests that the fluid-capsule system with small inertia is complicated and highly nonlinear. 


\section{Effect of initial tilted angle}

For both oblate and prolate spheroidal capsules, it is interesting to find that the long-term dynamics of a capsule can depend on the initial inclination angle in transitional regimes in the phase diagrams: at the same dimensionless shear rate, a capsule can adopt different modes of motion. For example in Fig. 4a, an oblate capsule adopts either a rolling or a precessing motion; even when $\beta$ falls into the range where a capsule undergoes a precessing motion, the average value of $\theta_{r y}$ (the cone angle of the trajectory of the capsule's revolution axis) still depends on the initial tilted angle $\beta$. We have observed a similar phenomenon for prolate capsules, which suggests the fluid-capsule system to be a high-dimensional dynamical system. In fact, the dependence of the final stable motion on the initial condition has been observed in other systems. Zhao and Shaqfeh [34] considered vesicles in shear flow with zero inertia, Huang et al. [51] studied prolate and oblate rigid particles in shear flow with finite inertia, and both reported off-the-shear-plane modes that are sensitive to the initial inclination angle of the vesicle or particle. These studies may shed some light on the intriguing phenomenon of red blood cells subjected to shear flow where individual cells adopt different types of motion even under the same flow condition [12].

\section{Reorientation}

We generalize the reorientation phenomenon discussed in Sec. III to both oblate and prolate capsules and a much wider range of dimensionless shear rates covering all modes of motion observed in the present study. Interestingly, for an oblate capsule, upon increasing the dimensionless shear rates, the unit vector (extending from the mass center of the capsule and pointing to the membrane material point that initially intersects with the revolution axis) changes from being aligned with the vorticity axis (in rolling motion) to being within the plane of shear (in tumbling motion), and finally turns back to being aligned with the vorticity axis (in the OS(R) mode). In between, the capsule adopts transitional off-the-shearplane motions (i.e., precessing and oscillating-swinging modes). It is also interesting to find the reorientation of the unit vector for a prolate capsule with increasing dimensionless shear rate is in an opposite order: the unit vector changes from being within the shear plane (in tumbling motion), to being aligned with the vorticity axis (in rolling motion), and finally changes back to being within the plane of shear. Similar to that of an oblate capsule, off-the-shear-plane modes are observed in between. Such a reorientation phenomenon and the difference between the reorientation of prolate and oblate spheroidal capsules can be utilized in applications such as particle alignment techniques.

\section{E. Effect of membrane constitutive laws and capsule aspect ratio}

Different membrane constitutive laws have been used in the present study: the strain-softening neo-Hookean $(\mathrm{NH})$ law and strain-hardening Skalaks (SK) law. When $C=1$ in Eq. (3), both constitutive laws lead to the same results at low dimensionless shear rates. We carried out simulations for an oblate capsule with an SK membrane with $C=1$, aspect ratio $2: 3$ and $\beta=45^{\circ}$. The phase diagram, corresponding to a horizontal line in Fig. 8, is the same to that of a capsule with an $\mathrm{NH}$ membrane. In the oscillating-swinging regime, we observe larger oscillating amplitudes of $\theta_{l x y}$ and $\theta_{l x z}$ for the capsule with an SK membrane. This is mainly because an SK membrane is strain-hardening and leads to less deformation at high shear rates when compared with an NH membrane. The effect of membrane constitutive laws has also been discussed by Dupont et al. [38]; they found that the main factor that determines the stable mode of motion and the transition is the deformation of the capsule instead of the membrane constitutive law. On these bases, we argue that the phase diagrams obtained in the present study are robust to membrane constitutive laws of the capsules.

We have also carried out simulations of capsules with higher sphericity. For an oblate capsule with an aspect ratio of $9: 10$, an $\mathrm{NH}$ membrane and an initial angle of $\beta=45^{\circ}$, we observe all types of motion and in the same order as in Fig. 8 when increasing the dimensionless shear rate. However, the upper boundary of $G$ for rolling motion is increased from 0.003 to 0.006 when compared with an oblate spheroidal capsule with aspect ratio of $2: 3$. When $G$ is further increased to 0.007 , the capsule is already in the oscillating-swinging regime. This suggests that the window of dimensionless shear rate for precessing and tumbling motions would be reduced when increasing the sphericity of an oblate spheroidal capsule. A similar phenomenon has been found for prolate capsules, the range of rolling motion for a prolate capsule with an aspect ratio of $3: 2$ and an SK membrane is $0.005 \leq G \leq 0.1$, slightly smaller than that of a capsule with aspect ratio of $2: 1$. In precessing motion at low dimensionless shear rates, we find the dependence of the precessing cone angle of the revolution axis on initial tilted angle is weaker for capsules with lower sphericity. For a prolate capsule with aspect ratio of $3: 2$, we observed that different values of $\beta$ can lead to precessing with different cone angles at the same dimensionless shear rate. However, for a capsule with an aspect ratio of $2: 1$, the cone angle seems only to be a function of the dimensionless shear rate for all cases considered. Finally, at high dimensionless shear rates where the capsule is undergoing the oscillating-swinging motion, we find the oscillation amplitudes of the angles $\theta_{l x y}$ and $\theta_{l x z}$ are smaller for a capsule of higher sphericity. 


\section{CONCLUSION}

We have studied the dynamics of oblate and prolate spheroidal capsules in simple shear flow with small inertia, for the case of equal internal and external fluid viscosity and without bending stiffness, using an immersed boundary lattice Boltzmann method. The effect of the initial tilted angle that the capsule's equatorial plane makes with the plane of shear on the motion the capsule would carry out after an initial transient stage has been investigated. Having identified the sequence of events when increasing the dimensionless shear rate, and characterized the various modes observed, form a necessary step to further experimental and theoretical work.

For oblate capsules, it is found here that tumbling, a previously well accepted mode of motion at low shear rates, is unstable. Instead, at very low dimensionless shear rates, independent of the initial angle, the capsule is found at late times to be in a rolling motion, wherein the capsule's equatorial plane tends to stay in the plane of shear. At larger dimensionless shear rates, a precessing motion is observed for most initial inclination angles over a long computational time. It is shown that this motion can be represented by Jeffery's theory [31] for a rigid spheroid in shear flow. Further increasing the shear rate leads to the well-known tumbling motion as the stable mode for a large range of inclination angles, in which the capsule flips with its revolution axis staying in the plane of shear. We find in transitional regimes, the capsule's motion could depend on the initial inclination angle. At high dimensionless shear rates, the capsule carries out a complicated oscillating-swinging motion: while the membrane is rotating around the liquid inside, the major axis of the capsule oscillates about the shear plane in addition to the previously found swinging about a mean angle with the flow direction. Both oscillations become weaker with the dimensionless shear rate, towards a steady tank treading motion asymptotically. The motion of a prolate spheroidal capsule is found to be different from that of an oblate one. When increasing the dimensionless shear rate, the stable modes are tumbling with the revolution axis staying in the plane of shear, precessing following Jeffery's orbit, log-rolling with revolution axis aligned with the vorticity axis, precessing with large deformation (wobbling), and finally oscillating-swinging at high dimensionless shear rates.

Both the rolling motion of an oblate capsule and the tumbling motion of a prolate capsule at very low dimensionless shear rates correspond to modes that contribute to increasing the particle shear stress. These are similar to the recent findings for rigid oblate and prolate solid particles in shear flow with small inertia [51], suggesting that the hypothesis of Jeffery [31] that non-spherical particles in Stokes shear flow finally adopt a motion with minimum energy dissipation does not apply to situations where inertial effects are significant. Finally, we have also reported here the interesting reorientation phenomenon, wherein modes are observed in opposite order for oblate and prolate capsule upon increasing the dimensionless shear rate, which can be used in particle alignment techniques.

\section{ACKNOWLEDGMENTS}

Z.W. is supported by a $\mathrm{PhD}$ studentship from Queen Mary University of London.
[1] T. M. Allen and P. R. Cullis, Science 303, 1818 (2004).

[2] V. P. Torchilin, Adv. Drug Deliv. Rev. (2012).

[3] J. B. Freund and B. Shapiro, Phys. Fluids 24, 051904 (2012).

[4] H. Lei, D. A. Fedosov, B. Caswell, and G. E. Karniadakis, J. Fluid Mech. 722, 214 (2013).

[5] D. Barthès-Biesel and J. M. Rallison, J. Fluid Mech. 113, 251 (1981).

[6] C. Pozrikidis, J. Fluid Mech. 297, 123 (1995).

[7] E. Lac, D. Barthès-Biesel, N. A. Pelekasis, and J. Tsamopoulos, J. Fluid Mech. 516, 303 (2004).

[8] E. Foessel, J. Walter, A.-V. Salsac, and D. BarthèsBiesel, J. Fluid Mech. 672, 477 (2011).

[9] D. Barthès-Biesel, Curr. Opin. Colloid Interface Sci. 16, 3 (2011).

[10] C. Misbah, Phys. Rev. Lett. 96, 028104 (2006).

[11] P. Bagchi and R. M. Kalluri, Phys. Rev. E 80, 016307 (2009).

[12] H. L. Goldsmith and J. Marlow, Proc. R. Soc. London B 182, 351 (1972).

[13] S. R. Keller and R. Skalak, J. Fluid Mech. 120, 27 (1982).

[14] S. Ramanujan and C. Pozrikidis, J. Fluid Mech. 361, 117
(1998)

[15] H. Noguchi and G. Gompper, Phys. Rev. Lett. 93, 258102 (2004).

[16] H. Noguchi and G. Gompper, Phys. Rev. E 72, 011901 (2005).

[17] D. A. Fedosov, B. Caswell, and G. E. Karniadakis, Biophys. J. 98, 2215 (2010).

[18] A. Yazdani and P. Bagchi, J. Fluid Mech. 718, 569 (2012).

[19] M. Abkarian, M. Faivre, and A. Viallat, Phys. Rev. Lett. 98, 188302 (2007).

[20] J. M. Skotheim and T. W. Secomb, Phys. Rev. Lett. 98, 078301 (2007).

[21] S. Kessler, R. Finken, and U. Seifert, J. Fluid Mech. 605, 207 (2008).

[22] Y. Sui, H. T. Low, Y. T. Chew, and P. Roy, Phys. Rev. E 77, 016310 (2008).

[23] Y. Sui, Y. T. Chew, P. Roy, Y. P. Cheng, and H. T. Low, Phys. Fluids 20, 112106 (2008).

[24] P. Bagchi and R. M. Kalluri, J. Fluid Mech. 669, 498 (2011).

[25] R. Finken, S. Kessler, and U. Seifert, J. Phys. Cond. 
Mat. 23, 184113 (2011).

[26] J. Walter, A.-V. Salsac, and D. Barthès-Biesel, J. Fluid Mech. 676, 318 (2011).

[27] W. R. Dodson III and P. Dimitrakopoulos, Phys. Rev. E 85, $021922(2012)$

[28] Y. Sui, Y. T. Chew, P. Roy, X. Chen, and H. T. Low, Phys. Rev. E 75, 066301 (2007).

[29] D.-V. Le, Phys. Rev. E 82, 016318 (2010).

[30] J. Dupire, M. Socol, and A. Viallat, Proc. Natl. Acad. Sci. USA 109, 20808 (2012).

[31] G. B. Jeffery, Proc. R. Soc. London A 102, 161 (1922).

[32] V. V. Lebedev, K. S. Turitsyn, and S. S. Vergeles, New J. Phys. 10, 043044 (2008).

[33] T. Biben, A. Farutin, and C. Misbah, Phys. Rev. E 83, 031921 (2011).

[34] H. Zhao and E. S. Shaqfeh, J. Fluid Mech. 674, 578 (2011).

[35] A. Farutin and C. Misbah, Phys. Rev. Lett. 109, 248106 (2012).

[36] H. Noguchi and G. Gompper, Phys. Rev. Lett. 98, 128103 (2007).

[37] P. M. Vlahovska, Y. N. Young, G. Danker, and C. Misbah, J. Fluid Mech. 678, 221 (2011).

[38] C. Dupont, A.-V. Salsac, and D. Barthès-Biesel, J. Fluid Mech. 721, 180 (2013).

[39] P. M. Vlahovska, D. Barthès-Biesel, and C. Misbah, C. R. Physique 14, 451 (2013).

[40] D. A. Fedosov, H. Noguchi, and G. Gompper, Biomech.
Model. Mechanobiol. in press (2013).

[41] X. Li, P. M. Vlahovska, and G. E. Karniadakis, Soft Matter 9, 28 (2013).

[42] M. Bitbol, Biophys. J. 49, 1055 (1986).

[43] Y. Sui, Y. T. Chew, P. Roy, and H. T. Low, J. Comput. Phys. 227, 6351 (2008).

[44] C. Dupont, A.-V. Salsac, D. Barthès-Biesel, M. Vidrascu, and L. T. Patrick, 11e Colloque National en Calcul des Structures, 13-17 May 2013, Giens, France, 1-8, CSMA2013.

[45] R. Skalak, A. Tozeren, R. P. Zarda, and S. Chien, Biophys. J. 13, 245 (1973).

[46] Y. Sui, Y. T. Chew, and H. T. Low, Int. J. Mod. Phys. C 18, 993 (2007).

[47] Y. Sui, Y. T. Chew, P. Roy, and H. T. Low, Comput. Fluids 38, 49 (2009).

[48] Y. Sui, X. Chen, Y. T. Chew, P. Roy, and H. T. Low, Comput. Fluids 39, 242 (2010).

[49] C. S. Peskin, J. Comput. Phys. 25, 220 (1977).

[50] D. Qi and L.-S. Luo, J. Fluid Mech. 477, 201 (2003).

[51] H. Huang, X. Yang, M. Krafczyk, and X.-Y. Lu, J. Fluid Mech. 692, 369 (2012).

[52] T. Omori, Y. Imai, T. Yamaguchi, and T. Ishikawa, Phys. Rev. Lett. 108, 138102 (2012).

[53] S. Kim and S. Karrila, Microhydrodynamics. 1991 (Butterworth-Heinemann, Boston).

[54] A. Karnis, H. Goldsmith, and S. G. Mason, Nature 200, 159 (1963). 